\title{
Research on Using IMRT Plan for Preoperative Rectal Cancer Patients
}

\author{
Quang Bui Vinh¹, Soai Dang Quoc ${ }^{2 *}$, Cuong Bui Xuan², Toan Hoang Van², Truong Vu² \\ ${ }^{1}$ Radiotherapy Department, Hanoi Oncology Hospital, Hanoi, Vietnam \\ ${ }^{2}$ Medicinal Supplies-Medical Physics Division, Hanoi Oncology Hospital, Hanoi, Vietnam \\ Email: quangbv@bvubhn.vn, *soaidq@bvubhn.vn,cuongbx@bvubhn.vn, \\ hoangtoan1586@gmail.com,vutruong29989@gmail.com
}

How to cite this paper: Bui Vinh, Q., Dang Quoc, S., Bui Xuan, C., Hoang Van, T. and Vu, T. (2020) Research on Using IMRT Plan for Preoperative Rectal Cancer Patients. International Journal of Medical Physics, Clinical Engineering and Radiation Oncology, 9, 165-177.

https://doi.org/10.4236/ijmpcero.2020.94015

Received: July 25, 2020

Accepted: October 9, 2020

Published: October 12, 2020

Copyright $\odot 2020$ by author(s) and Scientific Research Publishing Inc. This work is licensed under the Creative Commons Attribution International License (CC BY 4.0).

http://creativecommons.org/licenses/by/4.0/

\begin{abstract}
Aims: Research the possibility of using IMRT for rectal cancer patients in preoperative radiotherapy. Methods and Material: The research object is the preoperative radiotherapy plan for rectal cancer patients. The research group made two plans (IMRT, 3DCRT) for each image series of 34 rectal cancer patients who have received preoperative radiotherapy in Hanoi Oncology Hospital; and then compared the dose distribution on PTV, bladder, intestine, femoral bones, the average MU, and QA results of two types of plan. Results: The $95 \%$ isodose line and 50\% isodose of IMRT plan are closer than those of 3DCRT plan. The average dose of PTV in IMRT plan and 3DCRT plan are $5006 \pm 23 \mathrm{cGy}$ and $5036 \pm 42 \mathrm{cGy}$, respectively. The HTCI and HI values of IMRT and 3D plan are $0.97 \pm 0.026$ and $5.37 \pm 1.32 ; 1.00 \pm 0.003$ and $7.08 \pm$ 0.88 . About the dose of organ at risk: The maximum dose, average dose on the right, left femoral head in the IMRT plan are less than those values in the 3DCRT plan (6.2 Gy, $6 \mathrm{~Gy}, 7.4 \mathrm{~Gy}, 9 \mathrm{~Gy}$, respectively). The maximum dose and average dose on the bladder of the IMRT plan are smaller than those values of the 3DCRT plan (5.3 Gy, 1.5 times, respectively). The maximum dose and average dose of intestine in the IMRT plan was less than those values in the 3DCRT plan (4.3 Gy, 1.54 times, respectively). The MU number of IMRT plan is 1.5 times bigger than that of 3DCRT plan. Gamma index of IMRT plan is better than that of 3DCRT plan (99\% compared with 97\%). Conclusions: Using IMRT plan in preoperative radiotherapy for rectal cancer patients can still ensure covered PTV as well as the 3D PLAN. Furthermore, the dose of PTV in the IMRT plan is more uniform than those in the 3D plan, and the dose effect on the OAR surrounding PTV is much lower than when using the $3 \mathrm{D}$ plan. When IMRT plan were used to treat the preoperative rectal cancer patients, the LINAC took more time than when using 3DCRT plan.
\end{abstract}




\section{Keywords}

Rectal Cancer, Preoperative Radiotherapy, IMRT, HI, HTCI, Gamma Index

\section{Introduction}

According to the study of global cancer statistics 2018 by Freddie Bray et al., based on data from 185 countries, in the New Cases and Deaths for 36 Cancers and All Cancers Combined in 2018 Report, rectal cancer ranked the $9^{\text {th }}$ with 704,376 new cases and 31,394 deaths [1].

Generally, there have been many studies on IMRT radiation therapy for rectal cancer or cancer of pelvis region, such as the study of Yevgeniya Jane Mikhailovna Ioffe et al. In this research, eighty-three patients registered to be treated with IMRT. This study has shown the possible advantage of IMRT in improving long-term functioning of cervical cancer survivors, and in decreasing in pelvic girdle complications, which are likely to reduce pain levels and chronic morbidity and thus contribute to a higher psychosocial and sexual function. The vast majority of the patients in our study who experienced pelvic girdle complications post-RT were symptomatic with pain that became chronic or required the use of narcotic medications [2]-[16].

Another study by Rebecca M. Howell et al. found that Effective doses were higher for conventional radiotherapy compared to IMRT for all beam energies. IMRT significantly reduces dose to nearby organs, such as femoral heads, gonads, bladder, and colon, thereby yielding lower effective doses compared to the conventional radiotherapy [9]. Among the IMRT plans, plan with the $6 \mathrm{MV}$ beam energy results in the lowest effective dose. Salma K. Jabbour et al. also compared the acute toxicities of IMRT to 3D-conformal radiation therapy (3DCRT) in the treatment of rectal cancer, the research showed that IMRT reduces doses of the irradiated small bowel and IMRT can reduce median charges to small bowel by 5.1 Gy for rectal cancer [6].

According to the 2018 data of the World Health Organization (WHO), colorectal cancer is the fifth common cancer in Vietnam after cancer of the liver, lung, stomach and breast. Each year, there are nearly 15,000 new cases, equal the rate of $13.4 / 100,000$ people, and about more than 7000 deaths [17]. In recent years, accelerators with MLC have gradually become popular in Vietnam. Along with that, Intensity Modulated Radiation Therapy (IMRT) technology is also being progressively implemented in large hospitals. However, since most of the IMRT cases are concentrated for head and neck cancer, the deployment of the IMRT technique for pelvic cancer region has not been widely used in our hospital. Hence, this research is to evaluate and analyze to answer the following questions: Does the use of $6 \mathrm{MV}$ photon to plan IMRT for rectal cancer patients 
meet the requirements of the radiation treatment plan? Is there any difference between IMRT plan with 3DCRT plan using photon energy $6 \mathrm{MV}$ for the preoperative rectal cancer patients? Should we use IMRT plan for preoperative radiotherapy rectal cancer patients with $6 \mathrm{MV}$ photon? Do any immobilization equipment need when using IMRT plan for preoperative radiotherapy rectal cancer patients?

\section{Materials and Methods}

\section{Patients and simulation}

Thirty four patients with rectal cancer, subjected to radical preoperative radiotherapy were selected for this study. The research protocol was reviewed and approved by the Ethics Committee of Hanoi Oncology Hospital. All patients were simulated supine position with a full bladder. Computer tomography (CT) scans were acquired with $5 \mathrm{~mm}$ slice thickness through the L1 vertebral body to $5 \mathrm{~cm}$ below the perineum. The patients had urinated, drank a liter of water, and then waited for 30 minutes before being taken CT simulation [13] [14] [16] [17] [18].

\section{Target volume definition}

Target volumes were outlined on the planning CT scan by the treating radiation oncologist. The clinical target volume (CTV) was delineated according to published consensus guidelines [21]. The planning target volume (PTV) was defined with margins around the CTV of $0.5 \mathrm{~cm}$. The bladder, small bowel and femur heads were contoured as an organ at risk (OAR). The small bowel, bladder were outlined $0.5 \mathrm{~cm}$ outside of PTV, and femur heads were fully outlined. In addition, the healthy tissue was defined as the patient's volume included in the CT dataset minus $1.5 \mathrm{~cm}$ margin of the PTV volume.

\section{Dose constraints for PTV and OAR.}

Dose prescription for the PTV was 50.4 Gy in 1.8 Gy per fraction [6] [21]. Dose constraints for the PTV were as follows; 1 ) $\geq 100 \%$ of the PTV receives $\geq 95 \%$ of the prescribed dose, 2) PTV received 107\%. For OAR, small bowel V15 Gy $<120$ cc, max dose of small bowel $<115 \%$ dose prescription; max dose of bladder $<$ $115 \%$ of the dose prescription or $<50 \mathrm{~Gy}$, less than $10 \%$ of the volume of bladder $\geq 40 \mathrm{~Gy}$; max dose of femur heads $₫ 15 \%$ or $<50 \mathrm{~Gy}$, less than $10 \% \geq 40 \mathrm{~Gy}$.

\section{Planning techniques}

Two type of plans, 3D CRT, IMRT were created on the Eclipse Treatment Planning System (Version 13.5; Varian Medical Systems), and calculated with Anisotropic Analytical Algorithm (AAA), using a $2.5 \mathrm{~mm}$ calculation grid, using $6 \mathrm{MV}$ photon energy of Unique LINAC (that is LINAC made by VARIAN), a tissue heterogeneity correction was applied. The same dose constraint parameters of PTV and OAR were used for 3D CRT and IMRT planning [19] [20] [22] [23] [24] [25].

3D CRT planning: 3D CRT plans used four coplanar beams $\left(0^{\circ}, 90^{\circ}, 180^{\circ}\right.$, $270^{\circ}$ ) with a dose rate of $400 \mathrm{MU} / \mathrm{min}$ and beam energy of $6 \mathrm{MV}$ photons. 
IMRT planning: IMRT plans were optimized with Direct Machine parameter Optimization (DMPO) approach using eight coplanar beams $\left(215^{\circ}, 270^{\circ}, 295^{\circ}\right.$, $315^{\circ}, 45^{\circ}, 70^{\circ}, 150^{\circ}, 180^{\circ}$ ) with a dose rate of $400 \mathrm{MU} / \mathrm{min}$ and beam energy of 6-MV photons, sliding window technique.

Plan evaluation and comparison

Both of 3D CRT and IMRT plans were created verification plan. Then these plans were controlled quality by Delta 4 system, which is a product of Scandidos Company. The parameters of this system were set Dose Deviation (DD) 3\%; Distance to Agreement (DTA) 3 mm; Gamma Index (GI) 95\% [21].

Dosimetric parameters to analyze target coverage and dose distribution in the PTV are: 1) 3D max dose of plan ( $\left.D_{\max }\right)$; 2) max dose for PTV ( $\left.D_{\operatorname{maxPTV}}\right)$; 3 ) minimum dose of PTV ( $\left.\left.\mathrm{D}_{\text {mimPTV }}\right), 4\right)$ mean dose of PTV ( $\left.\mathrm{D}_{\text {meanPTV }}\right)$; the dose value cover $5 \%$ of PTV volume on Dose Volume Histogram $\left(\mathrm{D}_{5 \%}\right)$, the volume of PTV is covered with $95 \%$ of dose prescription $\left(\mathrm{V}_{95 \%}\right)$; the volume of PTV $\left(\mathrm{V}_{\mathrm{PTV}}\right)$. Homogeneity Index (HI) and HTCI Healthy Tissues Conformity Index (HTCI) were calculated with following formulas [23] [24] [25] [26].

$$
\mathrm{HI}=100 \% \times \frac{D_{5 \%}-D_{95 \%}}{D_{\text {pre }}}
$$

( $D_{\text {pre }}$ is the prescribed dose, $D_{5 \%}$ dose is corresponding to $5 \%$ of volume of PTV on the DVH $D_{95 \%}$ dose corresponding to 95 percent of volume of PTV on the DVH).

$$
\mathrm{HTCI}=\frac{V_{95 \%}}{V_{P T V}}
$$

The mean dose, maximum dose of bladder, small bowel, femoral heads, number of MU in both types of plan were selected, plotted on the graph. Probability density function was used to calculate the probability of the selected value.

\section{Results}

\section{1) Comparison of dose distribution on the transverse CT slice}

In Figure 1, observing the 95\% isodose line, that is the green line, in pairs of the sections (transversal, horizontal, vertical) of CT images of both IMRT and 3DCRT plans, the results showed that the isodose line of the IMRT plan bends and circles around the PTV line (the red line), and avoids the intestines (yellow line in the picture). In contrast, those of 3DCRT plan cover the PTV, and entire the intestine. Observing the lower isodose line, the $50 \%$ isodose line (the blue line) in the IMRT plan bends in PTV shape, and evades the intestine. However, the 3D CRT plan's dose is distributed in the entirebody in the direction of the beams, and it covers almost all the pelvis region.

2) Plan evaluation using Dose Volume Histogram ( DVH)

According to the Figure 2, the PTV dose in IMRT plan is similar to the PTV dose in 3DCRT plan. Meanwhile the Figure 3 and the Figure 4 show that the dose of OAR in IMRT plan is lower than that in 3DCRT plan. 


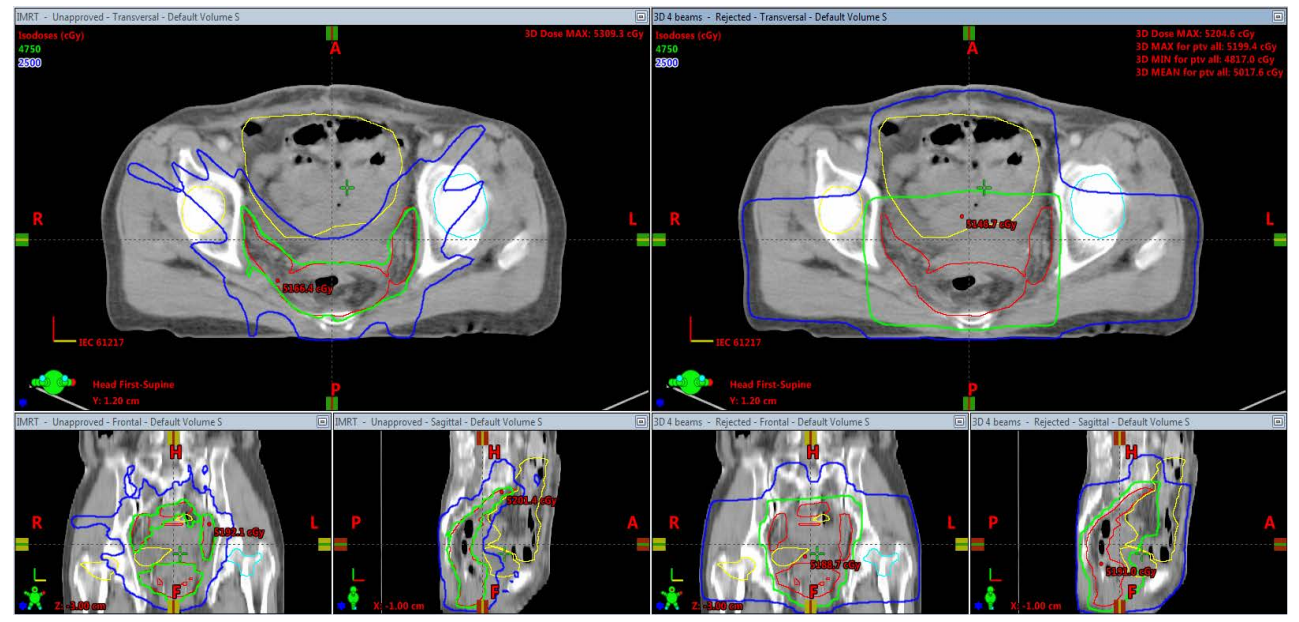

Figure 1. Compare dose distribution in the CT slice of IMRT plan (three left images) with those of 3DCRT plans (three right images). These were taken from Eclipse 13.5.

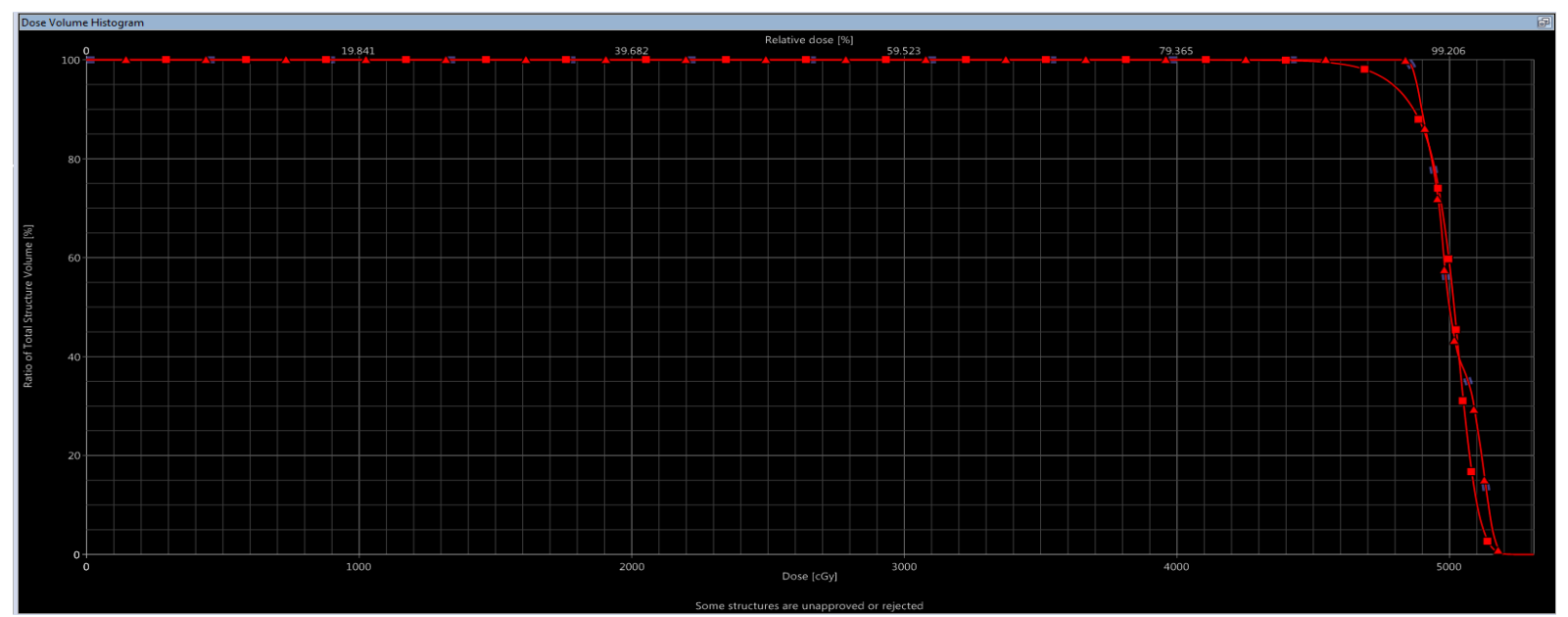

Figure 2. DVH of PTV in IMRT plan (red square dot line) and DVH of PTV in 3DCRT plan (red triangular dot line) taken from Eclipse 13.5.

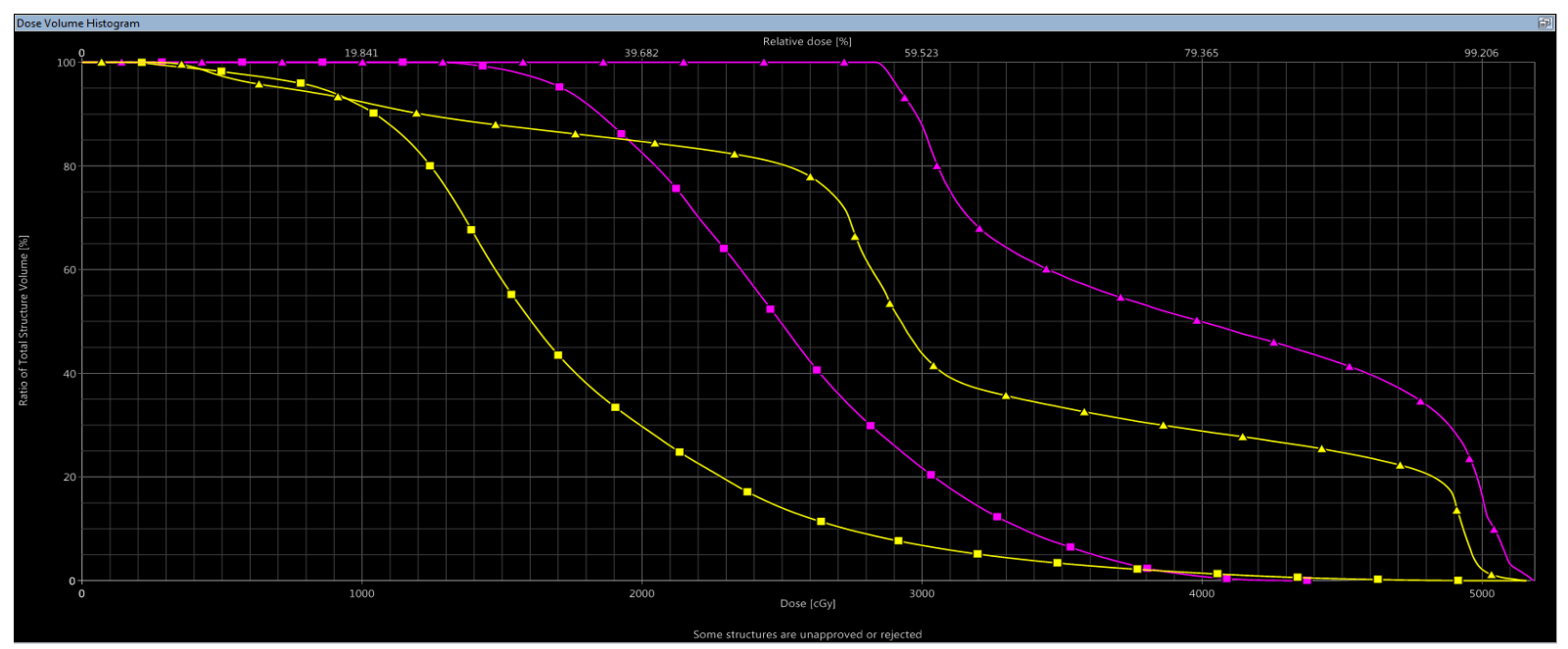

Figure 3. DVH of intestine (yellow line), and bladder (purple line) in IMRT plan (square dot line) and 3DCRT (triangular dot line) taken from Eclipse 13.5. 


\section{3) Dose of PTV, HI, HTCI}

Figure 5 shows that the max dose and mean dose of PTV in IMRT plan are similar to these in 3DCRT plan. However, the minimum dose of PTV in IMRT is lower than it in 3DCRT plan.

Figure 6 shows that the HTCI value of 3DCRT plan is equal one, and HTCI value of IMRT plan is almost equal one. Meanwhile, HI value of IMRT plan

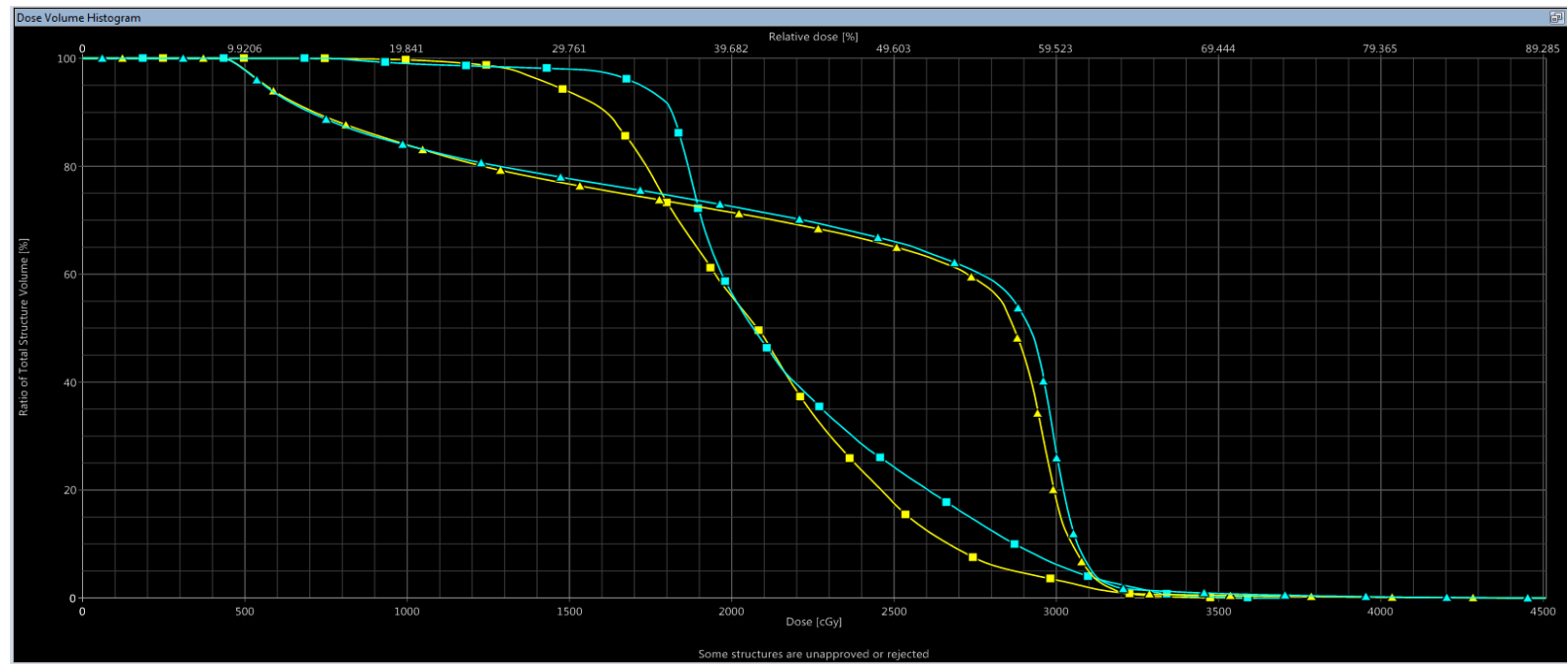

Figure 4. DVH of left femoral head (yellow line) and right femoral head (light green line) in IMRT plan (square dot line) and 3DCRT (triangular dot line) taken from Eclipse 13.5.

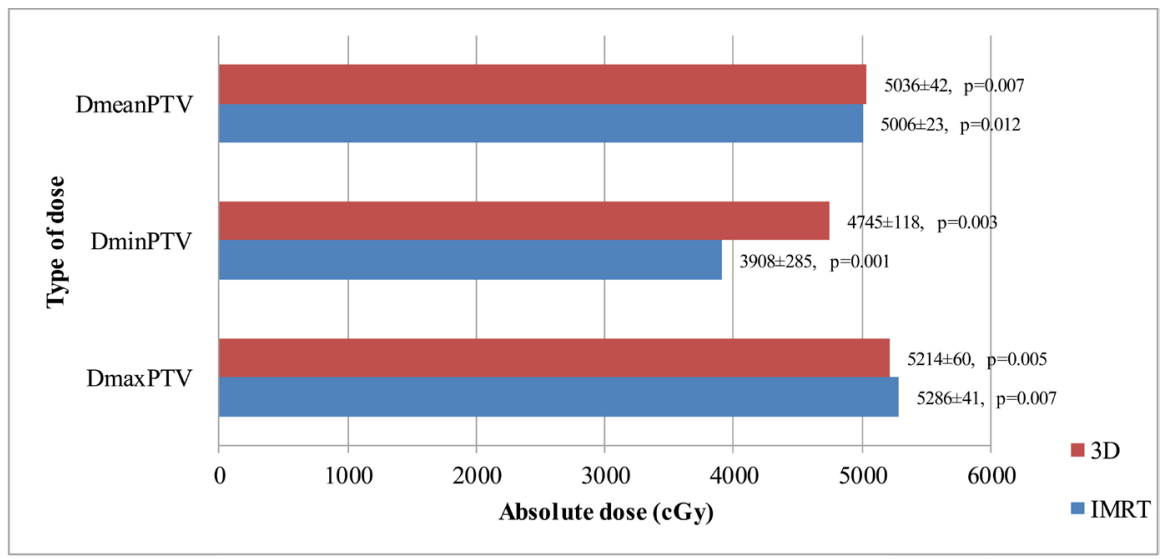

Figure 5. 3D max dose of PTV ( $\left.\mathrm{D}_{\text {maxPTV }}\right)$, 3D mean dose of PTV ( $\left.\mathrm{D}_{\text {meanPTV }}\right)$, 3D min dose of $\operatorname{PTV}\left(\mathrm{D}_{\min P T V}\right)$ in 3DCRT, and IMRT plan.

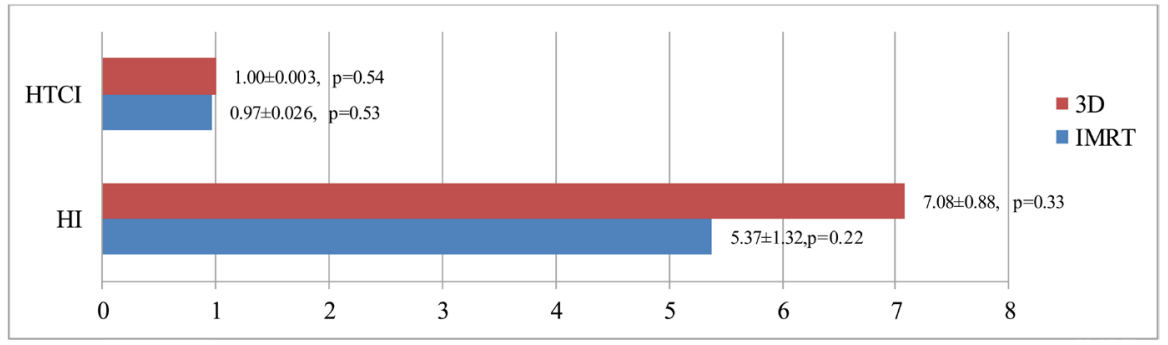

Figure 6. Average of HTCI, HI in IMRT, 3DCRT plan. 
is closer to 0 thanthat value of $3 \mathrm{DCRT}$ plan is.

4) The dose of Organ At Risk (OAR)

Figure 7 includes max dose of the intestine (DmaxI); mean dose of the intestine (DmeanI); Max dose of the bladder (DmaxB); Mean dose of the bladder (DmeanB); Max dose of the left femoral head (DmaxLF); Mean dose of the left femoral head (DmeanLF); Max dose of the right femoral head (DmaxRF); Mean dose of the right femoral head (DmeanRF). It shows that the maximum dose on the femoral head in both type of plan are smaller than $50 \mathrm{~Gy}$. The average dose is not more than $10 \%$ of the volume that femoral head receives, which is equal to and greater than $40 \mathrm{~Gy}$. The maximum dose on the bladder in the IMRT plan is smaller than $50 \mathrm{~Gy}$. The average dose on the bladder of the IMRT plan is smaller than $40 \mathrm{~Gy}$; those results meet the dose constraints. But the maximum and average doses of the bladder in the 3DCRT plan are bigger than $50 \mathrm{~Gy}$ and $40 \mathrm{~Gy}$, respectively, which do not meet the dose constraints. The intestinal dose of both types of plan do not meet the dose constraint for intestine in the above section.

Figure 8 shows that the intestinal volume receiving dose more than or equal

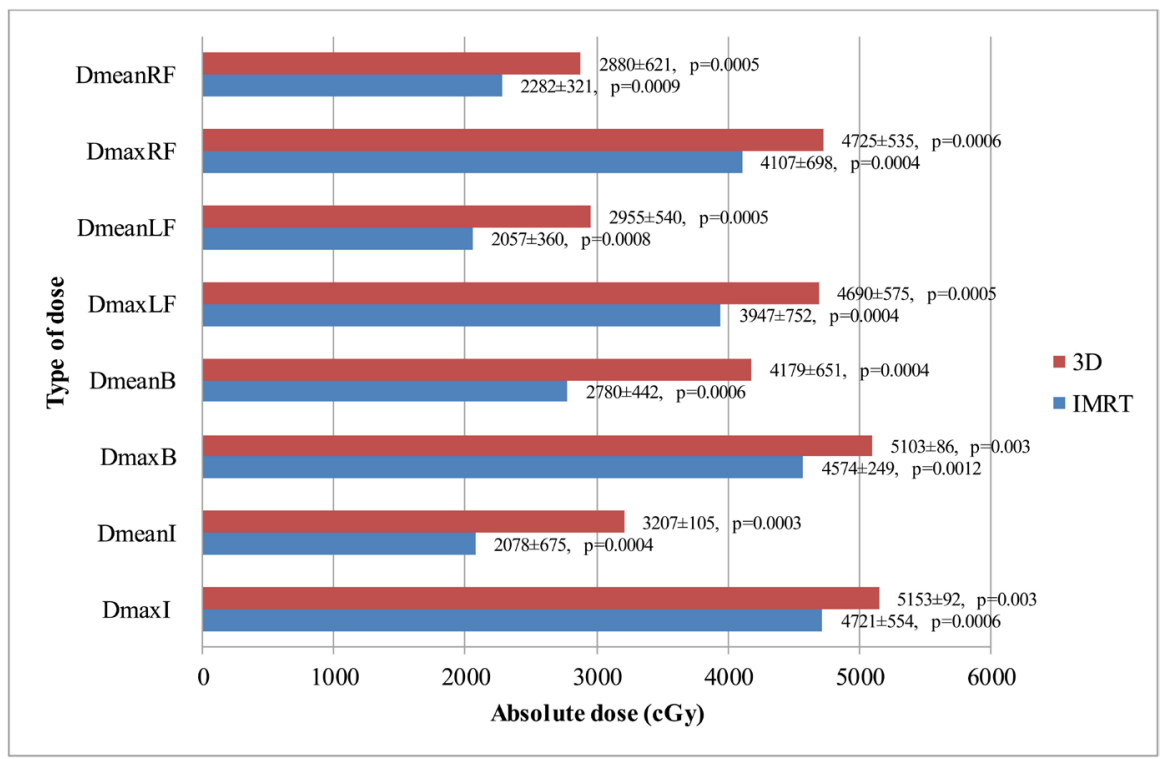

Figure 7. Absolute dose of organ at risk of IMRT plan and 3DCRT plan (Intestine, Bladder, and Femoral head).

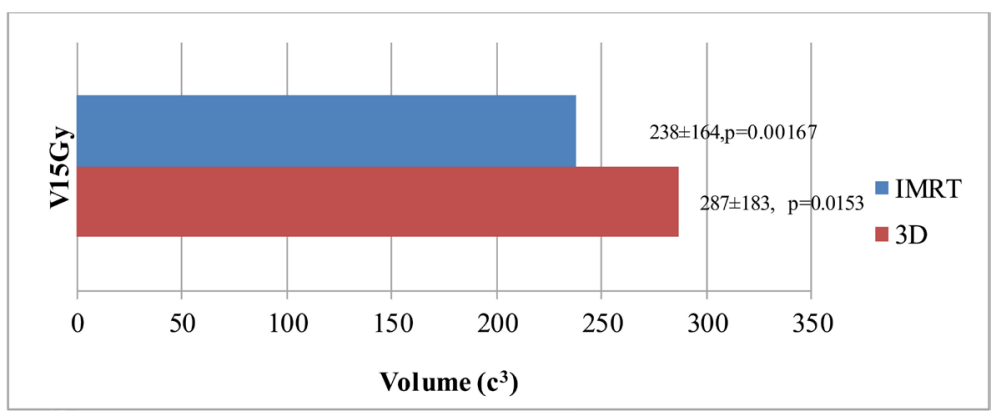

Figure 8. Intestinal volume receiving dose more than or equal to 15 Gy in IMRT plan and 3DCRT plan. 
to $15 \mathrm{~Gy}$ in IMRT plan is smaller than in 3DCRT plan $\left(238 \mathrm{c}^{3}\right.$ compared with $\left.287 c^{3}\right)$.

5) Result of a quality control plan, $M U$ number of plan

Figure 9 illustrates that gamma index of IMRT plan is better than that of 3DCRT plan (99\% versus $97 \%$ ). It means that $99 \%$ of measured points of IMRT plan passed. However, the passed measured points of 3DCRT are $97 \%$ of measured points.

Figure 10 shows that the number MU of IMRT was over five times higher than that of 3DCRT plan (1332 MU versus $258 \mathrm{MU}$ ).

\section{Discussion}

Dose distribution on the transverse CT slice: In the IMRT plan, medical physicists can flexibly alter the high dose distribution lines around PTV; It can change the high dose isodose line and the low dose isodose line close to or far away from PTV and OAR. Meanwhile, it is nearly impossible to create high dose and low dose isodose line curves along the PTV line and avoid OAR in the $3 \mathrm{D}$ plan. This dose distribution implies that the IMRT plan allows the minimization of the affected dose on OAR much better than the 3D plan does, while, at the same time it ensures the dose on PTV matching with prescribed dose.

Discussion of DVH results: The results on the top right corner of Figure 2 show that the minimum dose of PTV in the IMRT plan is lower than in the 3DCRT plan. The reason for this result is that the process of optimizing the IMRT plan that the authors have optimized the 95\% isodose line was closely covered PTV, so there were some PTV points had not been completely covered. However, as mentioned above, it is confirmed that $95 \%$ isodose line of the prescribed

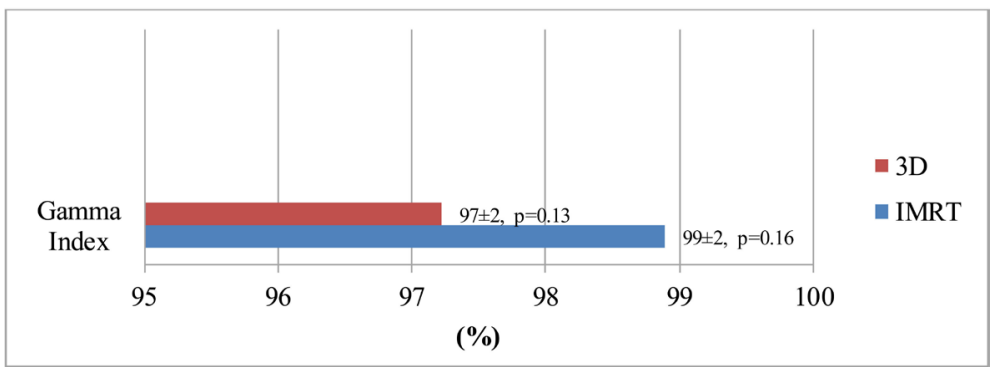

Figure 9. Gamma index of quality assurance plan of IMRT plan and 3DCRT plan.

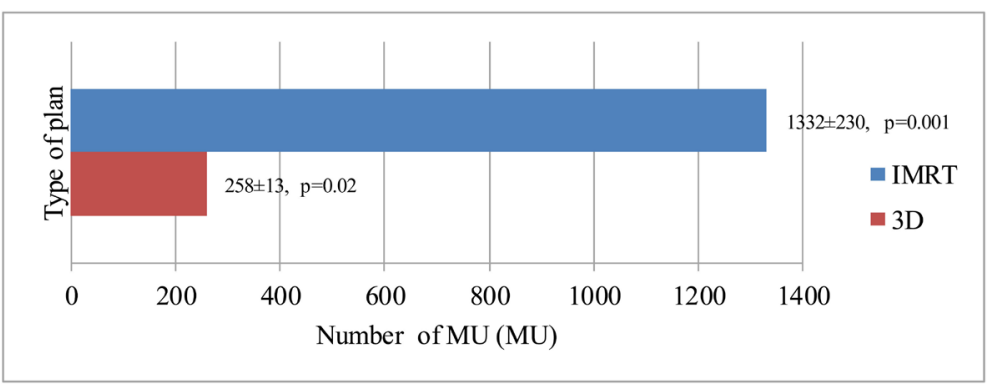

Figure 10. Average MU number of IMRT plan and 3DCRT plan. 
dose surrounding PTV can be changed dynamically in the IMRT plan. At the bottom right corner of Figure 2, it shows that the volume of PTV having dose more than prescribed dose was less than that of 3D CRT plan. The results in Figure 3 and Figure 4 confirmed again that IMRT plan conserved OAR better than 3DCRT plan, which is the same with the results in the research of Jun Shang et a and also other research [12]. In the research about comparing IMRT with 3DCRT in prostate treatment, Gary Luxton et al showed that the dose of bladder, bowel, femur in DVH of IMRT plan is better than those in 3DCRT plan [13].

The dose result on PTV: Quantitatively, Figure 5 shows that the max dose on both PTV in IMRT and 3DCRT plans were matched with the dose constraint for PTV (that must be less than $53.9 \mathrm{~Gy}$ ); and the average dose of PVT in both types of plan were similar with prescribed dose (50 Gy).

The HTCI value of the 3DCRT plan was 1; it means $100 \%$ of the PTV is covered by the $95 \%$ of prescribed dose. The HTCI of the IMRT plan was 0.97 , meaning that $97 \%$ of the PTV is covered by the $95 \%$ of prescribed dose. The IMRT plan should be re-optimized to increase to $100 \%$ of the PTV volume covered by $95 \%$ of prescribed dose.

The HI value of the IMRT plan was closer to zero than that value of the 3DCRT plan was. It means the difference between $\mathrm{D}_{95 \%}$ and $\mathrm{D}_{5 \%}$ of IMRT plan is smaller than that of 3DCRT plan. So the dose distribution in the PTV of the IMRT plan was more uniform than that of the 3DCRT plan. The reason is that the number of fields in the IMRT plan is over two time higher than that of 3DCRT plan, moreover the IMRT has intensity modulation during the bean on but 3DCRT did not have that. These reasons permit the IMRT plan avoid OAR better than 3DCRT plandoes.

The dose result on $O A R$ : The maximum dose, average dose on the right, left femoral head in the IMRT plan were less than those values of the 3DCRT plan (6.2 Gy, $6 \mathrm{~Gy}, 7.4 \mathrm{~Gy}, 9 \mathrm{~Gy}$, respectively). The maximum dose and average dose on the bladder of the IMRT plan were smaller than those values of the 3DCRT plan (5.3 Gy, 1.5 times, respectively). In the research "dosimetry and radiobiologic model comparison for IMRT and 3D conformal radiotherapy in treatment of carcinoma of the prostate" of Gary Luxton et al., IMRT plans have the mean dose that is smaller by $2.4 \mathrm{~Gy}$ (6.7\%) for bladder and $1.7 \mathrm{~Gy}(4.4 \%)$ for the rectum [13]. The maximum dose and average dose of the intestine in the IMRT plan were less than those values (4.3 Gy, 1.54 times, respectively). Moreover, the volume of intestine receiving dose equal to and greater than $15 \mathrm{~Gy}$ in the IMRT plan was smaller than that value of the 3D plan ( $238 c^{3}$ versus $\left.287 c^{3}\right)$.

The quality assurance result indicated that the difference between calculated dose and measured dose of the IMRT plan is smaller that that value of the 3DCRT plan. The MU number result showed that the MU number of the IMRT plan was 5.1 times higher than this number of the 3DCRT plan. It means that beam on time of the IMRT plan would be about 5 times larger than that of 
3DCRT plan. David Followill et al. also said IMRT normally requires a larger number of MU per treatment fraction related to conventional techniques [7]. The number MU of IMRT plan in this research is smaller than that of IMRT in David Followill's research (1332 MU comparing with 23,800 MU), both research used Varian MLC modulated. Therefore, ensuring the accuracy and safety for patients, when IMRT plan is used for preoperative radiotherapy, is extremely important. It confirms that using immobilization equipment during CT simulation and treatment, and quality assurance IMRT plan before using are essential.

\section{Conclusion}

The collected results and above data analysis prove that using IMRT plan in preoperative radiotherapy for rectal cancer patients can still ensure that PTV is covered as well as when using 3DCRT plan. Moreover, the dose distribution on PTV in the IMRT plan is more uniform than that in the 3DCRT plan, and the affection dose on OAR in the IMRT plan is lower than that in the 3DCRT plan. When using the IMRT plan in preoperative radiotherapy to treat rectal cancer, the LINAC takes five times longer to beam on than when using 3DCRT plan. It recommends that clinical centers should be equipped with immobilization and QA plan equipment.

\section{Acknowledgements}

The research facilities and manuscript submission are approved and sponsored by Hanoi Oncology Hospital, Vietnam. The authors would like to thank Prof. Huan Giap, Radiation Oncology Department, University of Miami Sylvester Cancer Center, USA and Prof. Michele Avanzo, Department of Medical Physics, CRO Centro di Riferimento Oncologico di Aviano, Italy for their invaluable comments that greatly improve this article. The authors also would like to thank Medicinal Supplies-Medical Physics Division and Radiotherapy Department, for supporting during the data collection process. The authors also would like to thank Dr. Nguyen Thanh Hang, International Cooperation and Scientific Research Unit, Hanoi Oncology Hospital, for editing English for this article.

\section{Conflicts of Interest}

The authors declare no conflicts of interest regarding the publication of this paper.

\section{References}

[1] Bray, F., Ferlay, F., Soerjomataram, I., Siegel, R.L., Torre, L.A. and Jemal, A. (2018) Global Cancer Statistics 2018: GLOBOCAN Estimates of Incidence and Mortality Worldwide for 36 Cancers in 185 Countries. CA: A Cancer Journal for Clinicians, 68, 394-424. https://doi.org/10.3322/caac.21492

[2] Hussein, M., Aldrige, S., Guerrero Urbano, T. and Nisbet, A. (2012) The Effect of 6 and $15 \mathrm{MV}$ on Intensity-Modulated Radiation Therapy Prostate Cancer Treatment: Plan Evaluation, Tumour Control Probability and Normal Tissue Complication 
Probability Analysis, and the Theoretial Risk of Secondary Induced Malignancies. The Bristish Journal of Radiology, 85, 423-432. https://doi.org/10.1259/bjr/24514638

[3] Arbea, L., Ramos, L.I., Martínez-Monge, R., Moreno, M. and Aristu, J. (2010) Intensity-Modulated Radiation Therapy (IMRT) vs.3D Conformal Radiotherapy (3DCRT) in Locally Advanced Rectal Cancer (LARC): Dosimetric Comparison and Clinical Implications. Radiation Oncology, 5, Article No. 17.

http://www.ro-journal.com/content/5/1/17

https://doi.org/10.1186/1748-717X-5-17

[4] Youssef, F.F., Parikh, P.J., DeWees, T.A., et al. (2016) Efficacy and Toxicity of Rectal Cancer Reirradiation Using IMRT for Patients Who Have Received Prior Pelvic Radiation Therapy. Advances in Radiation Oncology, 1, 94-100.

https://doi.org/10.1016/j.adro.2016.02.002

[5] Reyngold, M., Nilan, J., Veer, A.T., Bekaii-Saab, T., Lai, L., Meyer, J.E., et al. (2018) Trends in Intensity Modulated Radiation Therapy Use for Locally Advanced Rectal Cancer at National Comprehensive Cancer Network Centers. Advances in Radiation Oncology, 3, 34-41. https://doi.org/10.1016/j.adro.2017.10.001

[6] Jabbour, S.K., Patel, S., Herman, J.M., Wild, A., Nagda, S.N., Altoos, T.,et al. (2012) Intensity-Modulated Radiation Therapy for Rectal Carcinoma Can Reduce Treatment Breaks and Emergency Department Visists. International Journal of Surgical Oncology, 2012, Article ID: 891067. https://doi.org/10.1155/2012/891067

[7] Followill, D., Gee, P. and Boyer, A. (1997) Estimates of Whole-Body Dose Equivalent Froduced by Beam Intensity Modulated Confuramal Therapy. International Journal of Radiation Oncology, Biology, Physics, 38, 667-672. https://doi.org/10.1016/S0360-3016(97)00012-6

[8] Hall, E.J. and Wuu, C.-S. (2003) Radiation-Induced Second Cancers: The Impact of 3D-CRT. International Journal of Radiation Oncology, Biology, Physics, 56, 83-88. https://doi.org/10.1016/S0360-3016(03)00073-7

[9] Howell, R.M., Hertel, N.E., Wang, Z.L., Hutchinson, J. and Fullerton, G.D. (2006) Calculation of Effective Dose From Measurements of Secondary Neutron Spectra and Scattered Photon Dose From Dynamic MLC IMRT for $6 \mathrm{MV}, 15 \mathrm{MV}$, and 18 MV Beam Energies. American Association of Physicists in Medicine, 33, 360-368. https://doi.org/10.1118/1.2140119

[10] Mikhailovna Ioffe, Y.J., Hillen, T.J., Zhou, G.F., Schwarz, J.K., et al. (2014) Postradiation Damage to the Pelvic Girdle in Cervical Cancer Patients Is Intensity-Modulated Radiation Therapy Safer than Conventional Radiation? International Journal of Gynecological Cancer, 24, 806-812. https://doi.org/10.1097/IGC.0000000000000117

[11] Kry, S.F., Salehpour, M., Followill, D.S., Stovall, M., Kuban, D.A., Allen White, R. and Rosen, I.I. (2005) The Calculated Risk of Fatal Secondary Malignancies from Intensity Modulated Radiation Therapy. International Journal of Radiation Oncology, Biology, Physics, 62, 1195-1203. https://doi.org/10.1016/j.ijrobp.2005.03.053

[12] Kusters, M., Marijnen, C.A.M., Van De Velde, C.J.H., Rutten, H.J.T., Lahaye, M.J., Kim, J.H., Beets-Tan, R.G.H. and Beets, G.L. (2010) Patterns of Local Recurrence in Rectal Cancer; a Study of the Dutch TME Trial. EJSO, 36, 470-476. https://doi.org/10.1016/j.ejso.2009.11.011

[13] Luxton, G., Hancock, S.L. and Boyer, A.L. (2004) Dosimetry and Radiobiologic Model Comparison of IMRT and 3D Conformal Radiotherapy in Treatment of Carcinoma of the Prostate. International Journal of Radiation Oncology, Biology, Physics, 59, 267-284. https://doi.org/10.1016/j.ijrobp.2004.01.024 
[14] Shang, J., Kong, W. and Wang, Y.-Y., Ding, Z., Yan, G. and Zhe, H. (2014) VMAT Planning Study in Rectal Cancer Patients. Radiation Oncology, 9, Article No. 219. https://doi.org/10.1186/s13014-014-0219-1

[15] Vuong, T., Garant, A. and Gallant, F. (2014) Intensity-Modulated Radiation Therapy for Patients With Rectal Cancer. Colorectal Cancer, 3, 235-238.

https://doi.org/10.2217/crc.14.20

[16] Camma, C., Giunta, M., Fiorica, F., et al. (2000) Preoperative Radiotherapy for Resectable Rectal Cancer: A Meta-Analysis. JAMA, 284, 1008-1015. https://doi.org/10.1001/jama.284.8.1008

[17] http://benhvienk.vn/ung-thu-dai-truc-trang-ngay-cang-gia-tang-nd85399.html

[18] Mundt, A.J. and Roeske, J.C. (2002) Intensity-Modulated Radiation Therapy a Clinical Perspective. Seminars in Radiation Oncology, 12, 197-198.

[19] Hinckley, D. (1993) ICRU Report 50—Prescribing, Recording, and Reporting Photon Beam Therapy. Medical Physics, 21, 833-834.

[20] Varian Medical Systems, Inc. (2009) Eclipse Algorithms Reference Guide. P/N B502612R01A.

[21] Lee, N.Y., Riaz, N. and Lu, J.J. (2015) Target Volume Delineation for Conformal and Intensity-Modulated Radiation Therapy. Radiation Oncology, 303-314.

[22] Li, H., Dong, L., Zhang, L.F., Yang, J.N., Gillin, M.T. and Zhu, X.R. (2011) Toward A Better Understanding of the Gamma Index: Investigation of Parameters with Surface-Based Distance Method. Medical Physics, 38, 6730-6741. https://doi.org/10.1118/1.3659707

[23] Dang Quoc, S. (2016) Commissioning of the Analytical Anisotropic Algorithm (AAA) Calculation Algorithm for 2100 CD LINAC 18MV Photon Energy Beam. Master Thesis, Trieste University, Trieste.

[24] Dang Quoc, S., Bui Vinh, Q., Bui Xuan, C., Hoang Van, T. and Vu, T. (2019) Analyze the Dose Results Calculated with AAA Algorithm in Eclipse Software of New Treatment Planning System. Journal Pharmacy and Pharmacology, 7, 309-315. https://doi.org/10.4236/ijmpcero.2020.91001

[25] Dang Quoc, S., Bui Vinh, Q., Bui Xuan, C., Hoang Van, T. and Vu, T. (2020) Analyzing Planning Techniques for Whole Brain Radiotherapy. International Journal of Medical Physics, Clinical Engineering and Radiation Oncology, 9, 1-13. https://doi.org/10.4236/ijmpcero.2020.91001

[26] Kataria, T., Sharma, K., Subramani, V., Karrthick, K.P. and Bisht, S.S. (2012) Homogeneity Index: An Objective Tool for Assessment of Conformal Radiation Treatments. Journal of Medical Physics, 37, 207-213. https://doi.org/10.4103/0971-6203.103606 


\section{List of Abbreviations}

CT

$\mathrm{D}_{5 \%}$

$\mathrm{D}_{95 \%}$

DD

DmaxI

DmeanI

DmaxB

DmeanB

DmaxLF

DmeanLF

DmaxRF

DmeanRF

$\mathrm{D}_{\text {minPTV }}$

IMRT

3DCRT

DTA

DVH

$\mathrm{D}_{\operatorname{maxPTV}}$

$\mathrm{D}_{\text {meanPTV }}$

( $\left.\mathrm{D}_{\text {minPTV }}\right)$

$\mathrm{HI}$

HTCI

LINAC

MU

PTV

QA

$\mathrm{V}_{95 \%}$

$\mathrm{V}_{5 \%}$

$\mathrm{V}_{\text {PTV }}$

MLC
Computed Tomography

Dose corresponding to $5 \%$ of the volume of PTV on the DVH

Dose corresponding to 95 percent of the volume of PTV on the DVH

Dose Difference

Max Dose of the Intestine

Mean Dose of the Intestine

Max Dose of Bladder

Mean Dose of Bladder

Max Dose of the Left Femoral Head

Mean Dose of the Left Femoral Head

Max Dose of the Right Femoral Head

Mean Dose of the Right Femoral Head

3D min Dose of PTV

Intensity Modulated Radiation Therapy

Three Dimensional Conformal Radiotherapy

Distance to Agreement

Dose Volume Histograms

3D max Dose of PTV

3D Mean Dose of PTV

3D min Dose of PTV

Homogeneity Index

Healthy Tissues Conformity Index

Linear Accelerator

Monitor Unit

Planning Target Volume

Quality Assurance

Volume of PTV Corresponding to 95\% of Prescribed Dose on the DVH Volume of PTV Corresponding to 5\% of Prescribed Dose on the DVH

Volume of PTV

Multileaf Collimators 